\title{
Insect and Diseases Resistance in Tomato Entries
}

\author{
A. K. M. Quamruzzaman, Ferdouse Islam, Sharmilla Rani Mallick \\ Olericulture Division, Horticulture Research Center, Bangladesh Agricultural Research Institute, Gazipur, Bangladesh \\ Email: akmqzs@gmail.com
}

How to cite this paper: Quamruzzaman, A.K.M., Islam, F. and Mallick, S.R. (2021) Insect and Diseases Resistance in Tomato Entries. American Journal of Plant Sciences, $12,1646-1657$

https://doi.org/10.4236/ajps.2021.1211115

Received: September 25, 2021

Accepted: November 14, 2021

Published: November 17, 2021

Copyright (c) 2021 by author(s) and Scientific Research Publishing Inc. This work is licensed under the Creative Commons Attribution International License (CC BY 4.0).

http://creativecommons.org/licenses/by/4.0/ (c) (i) Open Access

\begin{abstract}
The study was conducted with 75 tomato entries at the farm of Olericulture Division, Horticulture Research Centre (HRC), Bangladesh Agricultural Research Institute (BARI), Gazipur, Bangladesh during the winter season of 2020-21 to evaluate insect and disease reaction. Among the various insect and diseases of tomatoes, the late blight, TYLCV, bacterial wilt infection and leaf miner, fruit borer infestation are most common in Bangladesh. The TYLCV infection was observed $0 \%$ to $27 \%$ infection, while 47 entries showed zero percent infection. The range of bacterial wilt infection was $0 \%$ to $10 \%$ and zero percent infection was observed in 62 entries. In case of leaf miner infestation and fruit borer infestation, the range was $0 \%$ to $43 \%$ and $0 \%$ to $10 \%$, respectively. Considering tolerance to late blight, TYLCV, bacterial wilt infection and leaf miner, fruit borer infestation, fruit size, fruit shape, plant growth nature, cluster nature of fruit, type of fruit ten entries AVTO 1010, AVTO 1706, AVTO 1713, AVTO 1829, AVTO 1909, AVTO 1911, AVTO 1915, AVTO 1921, AVTO 1954 and SLA 011 were found zero percent late blight, TYLCV, bacterial wilt infection and leaf miner, fruit borer infestation. So, these ten entries can be selected for disease and insect tolerant tomato varieties development as well as developing disease and insect tolerant hybrid tomato varieties.
\end{abstract}

\section{Keywords}

Insect, Diseases, Late Blight, TYLCV, Bacterial Wilt, Leaf Miner, Fruit Borer, Infestation, Resistance, Tomato

\section{Introduction}

Tomato (Solanum lycopersicum) is one of the most important vegetables of Bangladesh and the world too. The tomato is an important source of antioxi- 
dant. The essential phytonutrients found in the tomato fruit are lycopene, $\beta$-carotene, $\alpha$-tocopherol, polyphenols and ascorbic acid, and antioxidant activity depends on cultivar, environmental parameters, method of production and processing. The regular ingestion of an adequate amount of fresh tomatoes or processed tomato products has been inversely correlated with the development of widespread human diseases [1] [2] [3] and with an increase in plasma lipid peroxidation levels [4] [5]. This protective effect has been mainly attributed to the carotenoid constituents of the fruits, particularly lycopene and $\beta$-carotene which act as antioxidants in detoxifying free radicals [2] [6] [7] [8]. [9] reported that both fresh and processing tomatoes constitute significant amounts within produced vegetable crops.

Among the various insect and diseases of tomatoes, the late blight, TYLCV (Tomato yellow leaf curl virus), bacterial wilt infection and leaf miner, fruit borer infestation are most common in Bangladesh. The polyphagous nature of different insects, and development of insecticide resistance, provide challenges to effective management. Resistance in tomato is important for management of fruit borer. The highest levels of arthropod resistance are widely reported in wild varieties such as L. hirsutum Dunal. [10]. Attempts have been made to identify sources of resistance in L. esculentum [11] [12]. Breeding tomatoes for insect resistance is generally limited to identification of sources and mechanisms of resistance. Emphasis should be placed on development of commercial insect and disease resistant cultivars with desirable yield and market attributes [13].

Heavy use of insecticides to control these pests has resulted in the development of resistance in some pest populations [14], enhancement of the destructiveness of the leaf miner due to loss of its natural enemies [15], and may also pose environmental problems. Morphological and physical characteristics of plants are associated with attraction, feeding, and oviposition of insect pests [16]. Thus, plant phenology may influence pest populations because of factors such as maturation date, which vary according to plant cultivar. In addition, pest population development is favored by high temperatures and plant cultivars that vary in their development time may experience differing levels of pest pressure [17]. Under field conditions, temperature and relative humidity have been shown to affect populations of tomato pests such as aphids (Aphidoidea) and whiteflies (Aleyrodidae) [18].

Therefore, development and deployment of cultivars with resistance to the important insect pests of tomato are needed. However, Bangladesh Agricultural Research Institute (BARI) has released varieties that are higher yielder, but some varieties are prone to several pests and diseases. Beside this growers' demand is good quality hybrid varieties having tolerance to different major insects and diseases. In this context, BARI needs to be developed tomato varieties possess traits of virus and wilt resistance. In 2020, The World Vegetable Centre has supplied 43 tomato advanced entries to BARI. This study was undertaken to screen tomato germplasm from AFACI supplied germplasm, advanced varieties, cultivars, and native entries for resistance against late blight, TYLCV, bacterial wilt infec- 
tion and leaf miner, fruit borer infestation and for other morphological attributes in order to develop insect, diseases resistant cultivars.

\section{Materials and Methods}

\subsection{Experimental Site}

The experiment was conducted at the Olericulture Division of Horticulture Research Centre, Bangladesh Agricultural Research Institute (BARI) during $2020-21$. The experimental field was at $23.9920^{\circ} \mathrm{N}$ Latitude and $90.4125^{\circ} \mathrm{E}$ Longitudes having an elevation of $8.2 \mathrm{~m}$ from sea level under agro-ecological zone (AEZ) 28. The average minimum and maximum temperature were $18.0^{\circ} \mathrm{C}$ and $29.0^{\circ} \mathrm{C}$ and the average relative humidity was $58.2 \%$. The soil of the experimental field was sandy clay loam in texture having a $\mathrm{pH}$ range around 6.0.

Air temperatures and relative humidity of the experimental area

Average monthly minimum air temperatures, maximum air temperatures and average relative humidity during the season are shown in Table 1 .

\subsection{Plant Materials}

Seventy-five tomato entries (WorldVeg Center: 41 nos., BARI varieties: 10 nos., local germplasm 17 nos., exotic germplasm 7 nos.) were included in the study (Table 2). The seeds were sown on the seedbed on 01 October 2020. Thirty-two days old seedlings were transplanted in the main field on 01 November, 2020.

\subsection{Experimental Design and Layout}

The experiment was laid out in an RCB design with three replications. The plot size was $5.0 \times 1.0 \mathrm{~m}$ where 20 plants were planted with space of $60 \times 50 \mathrm{~cm}$ in two rows.

\subsection{Land Fertilization}

The experimental area was enriched with organic fertilizer, Nitrogen, Phosphorus, Potassium, Sulphur, Zinc and Boron @ 3000, 250, 90, 125, 20, 3 and 2 kg/ha, correspondingly. One third of the organic fertilizer, $50 \%$ of Phosphorus and full of Sulphur, Zinc and Boron were incorporated for the period of last land-dwelling

Table 1. Average monthly minimum temperature $\left({ }^{\circ} \mathrm{C}\right)$, maximum temperature $\left({ }^{\circ} \mathrm{C}\right)$ and relative humidity (\%) recorded during winter season.

\begin{tabular}{cccccccc}
\hline Parameter & $\begin{array}{c}\text { Oct } \\
2020\end{array}$ & $\begin{array}{c}\text { Nov } \\
2020\end{array}$ & $\begin{array}{c}\text { Dec } \\
2020\end{array}$ & $\begin{array}{c}\text { Jan } \\
2021\end{array}$ & $\begin{array}{c}\text { Feb } \\
2021\end{array}$ & $\begin{array}{c}\text { Mar } \\
2021\end{array}$ & Mean \\
\hline $\begin{array}{c}\text { Monthly minimum air } \\
\text { temperature }\left({ }^{\circ} \mathrm{C}\right)\end{array}$ & 24 & 19 & 14 & 16 & 15 & 20 & 18.0 \\
$\begin{array}{c}\text { Monthly maximum air } \\
\text { temperature }\left({ }^{\circ} \mathrm{C}\right)\end{array}$ & 31 & 29 & 26 & 28 & 28 & 32 & 29.0 \\
$\begin{array}{c}\text { Monthly mean relative } \\
\text { humidity }(\%)\end{array}$ & 72 & 66 & 63 & 54 & 49 & 45 & 58.2 \\
\hline
\end{tabular}


Table 2. Seventy-five tomato entries.

\begin{tabular}{|c|c|c|c|c|c|}
\hline \multicolumn{3}{|c|}{ WorldVeg Center germplasm } & \multirow{2}{*}{$\begin{array}{l}\text { BARI varieties } \\
\text { BARI Tomato-2 }\end{array}$} & \multirow{2}{*}{$\begin{array}{c}\begin{array}{c}\text { Local } \\
\text { germplasm }\end{array} \\
\text { SLA } 011\end{array}$} & \multirow{2}{*}{$\begin{array}{c}\begin{array}{c}\text { Exotic } \\
\text { germplasm }\end{array} \\
\text { SLA } 004\end{array}$} \\
\hline AVTO 0301 & AVTO 1706 & AVTO 1911 & & & \\
\hline AVTO 1003 & AVTO 1707 & AVTO 1913 & BARI Tomato-11 & SLA 012 & SLA 005 \\
\hline AVTO 1008 & AVTO 1711 & AVTO 1914 & BARI Tomato-14 & SLA 013 & SLA 006 \\
\hline AVTO 1010 & AVTO 1712 & AVTO 1915 & BARI Tomato-15 & SLA 014 & SLA 007 \\
\hline AVTO 1219 & AVTO 1713 & AVTO 1919 & BARI Tomato-16 & SLA $015-1$ & SLA 008 \\
\hline AVTO 1288 & AVTO 1715 & AVTO 1921 & BARI Tomato-17 & SLA 015-2 & SLA 009 \\
\hline AVTO 1306 & AVTO 1716 & AVTO 1954 & BARI Tomato-18 & SLA 015-3 & SLA 010 \\
\hline AVTO 1314 & AVTO 1717 & & BARI Tomato-19 & SLA 018 & \\
\hline AVTO 1315 & AVTO 1718 & & BARI Tomato 20 & SLA $025-1$ & \\
\hline AVTO 1409 & AVTO 1719 & & BARI Tomato 21 & SLA $025-2$ & \\
\hline AVTO 1424 & AVTO 1720 & & & SLA $025-3$ & \\
\hline AVTO 1429 & AVTO 1828 & & & SLA $025-4$ & \\
\hline AVTO 1464 & AVTO 1829 & & & SLA $025-5$ & \\
\hline AVTO 1616 & AVTO 1903 & & & SLA 025-6 & \\
\hline AVTO 1619 & AVTO 1907 & & & SLA 025-7 & \\
\hline AVTO 1702 & AVTO 1909 & & & FOBHT8 & \\
\hline AVTO 1705 & AVTO 1910 & & & MOBHT8 & \\
\hline & 41 & & 10 & 17 & 7 \\
\hline
\end{tabular}

preparation. Rest of organic fertilizer and Phosphorus and 1/3 of Potassium were applied as basal in pit. Entire quantity of Nitrogen and rest of Potassium were applied in four equal portions beginning from 20 days after transplanting. Rest three portions were fertilized at 20,40 and 60 days after transplanting.

\subsection{Data Recorded}

Data on yield and yield attributing parameters with qualitative traits were recorded from 20 inner plants of each plot escaping border plants following World Veg guideline. Tomato fruits were started harvesting at breaker stage from 20 inner plant of each treatment. Four to six harvesting was done according to the different germplasm characters and data on late blight infection (\%), TYLCV infection (\%), bacterial wilt infection (\%), leaf miner infestation (\%), fruit borer infestation (\%), fruit size, fruit shape, plant growth nature, cluster nature of fruit, type of fruit.

\section{Results and Discussion}

The tomato entries differed significantly in all parameters studied and the results have been shown in Table 3 and Table 4. In respect of pest and disease reaction, 
Table 3. Late blight, TYLCV, bacterial wilt infection and leaf miner, fruit borer infestation of 75 tomato entries.

\begin{tabular}{|c|c|c|c|c|c|}
\hline Entries & $\begin{array}{l}\text { Late blight } \\
\text { infection } \\
(\%)\end{array}$ & $\begin{array}{l}\text { TYLCV } \\
\text { infection } \\
(\%)\end{array}$ & $\begin{array}{l}\text { Bacterial wilt } \\
\text { infection } \\
(\%)\end{array}$ & $\begin{array}{l}\text { Leaf miner } \\
\text { infestation } \\
\quad(\%)\end{array}$ & $\begin{array}{c}\text { Fruit borer } \\
\text { infestation } \\
(\%)\end{array}$ \\
\hline AVTO 0301 & 50 & 13 & 10 & 17 & 10 \\
\hline AVTO 1003 & 0 & 10 & 0 & 0 & 3 \\
\hline AVTO 1008 & 0 & 10 & 0 & 0 & 7 \\
\hline AVTO 1010 & 0 & 0 & 0 & 0 & 0 \\
\hline AVTO 1219 & 27 & 27 & 7 & 20 & 7 \\
\hline AVTO 1288 & 0 & 23 & 0 & 0 & 0 \\
\hline AVTO 1306 & 47 & 23 & 0 & 27 & 0 \\
\hline AVTO 1314 & 27 & 0 & 0 & 40 & 0 \\
\hline AVTO 1315 & 53 & 0 & 0 & 20 & 0 \\
\hline AVTO 1409 & 50 & 0 & 0 & 23 & 0 \\
\hline AVTO 1424 & 37 & 0 & 0 & 43 & 0 \\
\hline AVTO 1429 & 0 & 17 & 0 & 0 & 0 \\
\hline AVTO 1464 & 43 & 20 & 7 & 37 & 7 \\
\hline AVTO 1616 & 37 & 0 & 0 & 27 & 0 \\
\hline AVTO 1619 & 0 & 0 & 0 & 0 & 7 \\
\hline AVTO 1702 & 27 & 20 & 0 & 23 & 7 \\
\hline AVTO 1705 & 23 & 0 & 0 & 23 & 0 \\
\hline AVTO 1706 & 0 & 0 & 0 & 0 & 0 \\
\hline AVTO 1707 & 50 & 20 & 0 & 40 & 7 \\
\hline AVTO 1711 & 0 & 0 & 0 & 0 & 3 \\
\hline AVTO 1712 & 40 & 0 & 0 & 7 & 7 \\
\hline AVTO 1713 & 0 & 0 & 0 & 0 & 0 \\
\hline AVTO 1715 & 50 & 0 & 0 & 23 & 0 \\
\hline AVTO 1716 & 43 & 23 & 10 & 30 & 0 \\
\hline AVTO 1717 & 43 & 0 & 0 & 7 & 7 \\
\hline AVTO 1718 & 53 & 0 & 0 & 23 & 7 \\
\hline AVTO 1719 & 53 & 0 & 0 & 40 & 0 \\
\hline AVTO 1720 & 47 & 10 & 0 & 37 & 7 \\
\hline AVTO 1828 & 0 & 17 & 0 & 0 & 0 \\
\hline AVTO 1829 & 0 & 0 & 0 & 0 & 0 \\
\hline AVTO 1903 & 0 & 0 & 0 & 0 & 7 \\
\hline
\end{tabular}




\section{Continued}

\begin{tabular}{|c|c|c|c|c|c|}
\hline AVTO 1907 & 0 & 0 & 0 & 0 & 0 \\
\hline AVTO 1909 & 0 & 0 & 0 & 0 & 0 \\
\hline AVTO 1910 & 0 & 13 & 10 & 0 & 7 \\
\hline AVTO 1911 & 0 & 0 & 0 & 0 & 0 \\
\hline AVTO 1913 & 0 & 17 & 0 & 0 & 7 \\
\hline AVTO 1914 & 0 & 0 & 0 & 0 & 7 \\
\hline AVTO 1915 & 0 & 0 & 0 & 0 & 0 \\
\hline AVTO 1919 & 50 & 0 & 0 & 40 & 0 \\
\hline AVTO 1921 & 0 & 0 & 0 & 0 & 0 \\
\hline AVTO 1954 & 0 & 0 & 0 & 0 & 0 \\
\hline SLA 004 & 47 & 20 & 0 & 23 & 7 \\
\hline SLA 005 & 33 & 20 & 0 & 23 & 7 \\
\hline SLA 006 & 50 & 0 & 0 & 23 & 3 \\
\hline SLA 007 & 43 & 0 & 0 & 7 & 0 \\
\hline SLA 008 & 50 & 0 & 0 & 23 & 0 \\
\hline SLA 009 & 47 & 20 & 10 & 20 & 7 \\
\hline SLA 010 & 23 & 0 & 0 & 27 & 3 \\
\hline SLA 011 & 0 & 0 & 0 & 0 & 0 \\
\hline SLA 012 & 20 & 0 & 0 & 20 & 3 \\
\hline SLA 013 & 0 & 13 & 7 & 27 & 7 \\
\hline SLA 014 & 23 & 3 & 0 & 23 & 0 \\
\hline SLA 015-1 & 23 & 7 & 7 & 17 & 0 \\
\hline SLA $015-2$ & 40 & 7 & 0 & 23 & 7 \\
\hline SLA 015-3 & 23 & 0 & 3 & 20 & 0 \\
\hline SLA 018 & 27 & 23 & 10 & 0 & 0 \\
\hline SLA 025-1 & 23 & 0 & 7 & 37 & 0 \\
\hline SLA 025-2 & 0 & 7 & 7 & 3 & 0 \\
\hline SLA 025-3 & 0 & 0 & 0 & 37 & 0 \\
\hline SLA 025-4 & 23 & 7 & 0 & 0 & 7 \\
\hline SLA 025-5 & 23 & 0 & 0 & 27 & 0 \\
\hline SLA 025-6 & 0 & 0 & 0 & 7 & 0 \\
\hline SLA 025-7 & 0 & 0 & 0 & 37 & 0 \\
\hline FOBHT8 & 0 & 0 & 0 & 20 & 10 \\
\hline МОВНТ8 & 0 & 0 & 0 & 7 & 10 \\
\hline
\end{tabular}




\begin{tabular}{cccccc} 
Continued & & & & \\
\hline BARI Tomato-2 & 23 & 0 & 0 & 23 & 0 \\
BARI Tomato-11 & 0 & 0 & 0 & 7 & 0 \\
BARI Tomato-14 & 23 & 20 & 0 & 7 & 7 \\
BARI Tomato-15 & 23 & 7 & 0 & 20 & 7 \\
BARI Tomato-16 & 0 & 0 & 0 & 20 & 0 \\
BARI Tomato-17 & 37 & 0 & 0 & 23 & 7 \\
BARI Tomato-18 & 0 & 0 & 0 & 20 & 0 \\
BARI Tomato-19 & 0 & 0 & 0 & 0 & 7 \\
BARI Tomato 20 & 20 & 3 & 0 & 0 & 0 \\
BARI Tomato 21 & 0 & 0 & 0 & 20 & 0 \\
Range & $0-53$ & $0-27$ & $0-10$ & $0-43$ & $0-10$ \\
Mean & 20.0 & 5.6 & 1.3 & 15.2 & 2.8 \\
\hline
\end{tabular}

Table 4. Fruit size, fruit shape, plant growth nature, cluster nature of fruit, type of fruit characters of 75 tomato entries.

\begin{tabular}{|c|c|c|c|c|c|}
\hline Entries & Fruit size & Fruit shape & $\begin{array}{c}\text { Plant } \\
\text { growth } \\
\text { nature }\end{array}$ & $\begin{array}{l}\text { Cluster } \\
\text { nature } \\
\text { of fruit }\end{array}$ & Type of fruit \\
\hline AVTO 0301 & Medium & Round & Determinate & Cluster & Table purpose \\
\hline AVTO 1003 & Medium & Oval & Determinate & Cluster & Table purpose \\
\hline AVTO 1008 & Medium & Round & Determinate & Cluster & Table purpose \\
\hline AVTO 1010 & Medium & Round & Determinate & Cluster & Table purpose \\
\hline AVTO 1219 & Medium & Oval & Determinate & Cluster & Table purpose \\
\hline AVTO 1288 & Medium & Oval & Determinate & Cluster & Table purpose \\
\hline AVTO 1306 & Medium & Round & Determinate & Cluster & Table purpose \\
\hline AVTO 1314 & Medium & Flat round & Determinate & Cluster & Table purpose \\
\hline AVTO 1315 & Medium & Round & Determinate & Cluster & Table purpose \\
\hline AVTO 1409 & Medium & Round & Determinate & Cluster & Table purpose \\
\hline AVTO 1424 & Medium & Round & Determinate & Cluster & Table purpose \\
\hline AVTO 1429 & Medium & Round & Indeterminate & Cluster & Cherry type \\
\hline AVTO 1464 & Small & Round & Indeterminate & Cluster & Cherry type \\
\hline AVTO 1616 & Medium & Round & Semi Determinate & Cluster & Table purpose \\
\hline AVTO 1619 & Medium & Oval & Determinate & Cluster & Table purpose \\
\hline AVTO 1702 & Medium & Round & Determinate & Cluster & Table purpose \\
\hline AVTO 1705 & Medium & Oval & Determinate & Cluster & Table purpose \\
\hline AVTO 1706 & Medium & Round & Determinate & Cluster & Table purpose \\
\hline
\end{tabular}




\section{Continued}

\begin{tabular}{|c|c|c|c|c|c|}
\hline AVTO 1707 & Medium & Oval & Determinate & Cluster & Table purpose \\
\hline AVTO 1711 & Big & Flat round & Determinate & Cluster & Table purpose \\
\hline AVTO 1712 & Medium & Flat round & Determinate & Cluster & Table purpose \\
\hline AVTO 1713 & Big & Flat round & Determinate & Cluster & Table purpose \\
\hline AVTO 1715 & Big & Flat round & Determinate & Cluster & Table purpose \\
\hline AVTO 1716 & Medium & Flat round & Determinate & Cluster & Table purpose \\
\hline AVTO 1717 & Big & Flat round & Determinate & Cluster & Table purpose \\
\hline AVTO 1718 & Big & Flat round & Determinate & Cluster & Table purpose \\
\hline AVTO 1719 & Medium & Round & Determinate & Cluster & Table purpose \\
\hline AVTO 1720 & Big & Flat round & Determinate & Cluster & Table purpose \\
\hline AVTO 1828 & Medium & Round & Indeterminate & Cluster & Cherry type \\
\hline AVTO 1829 & Medium & Oval & Indeterminate & $\begin{array}{c}\text { Cluster } \\
\text { (long) }\end{array}$ & Cherry type \\
\hline AVTO 1903 & Medium & Round & Determinate & Cluster & Table purpose \\
\hline AVTO 1907 & Medium & Oval & Determinate & $\begin{array}{c}\text { Cluster } \\
\text { (long) }\end{array}$ & Table purpose \\
\hline AVTO 1909 & Medium & Oval & Semi Determinate & Cluster & Table purpose \\
\hline AVTO 1910 & Medium & Round & Semi Determinate & Cluster & Table purpose \\
\hline AVTO 1911 & Medium & Round & Determinate & Cluster & Table purpose \\
\hline AVTO 1913 & Medium & Oval & Determinate & Cluster & Table purpose \\
\hline AVTO 1914 & Medium & Oval & Determinate & Cluster & Table purpose \\
\hline AVTO 1915 & Medium & Oval & Semi Determinate & Cluster & Table purpose \\
\hline AVTO 1919 & Medium & Round & Determinate & Cluster & Table purpose \\
\hline AVTO 1921 & Medium & Round & Determinate & Cluster & Table purpose \\
\hline AVTO 1954 & Medium & Round & Determinate & Cluster & Table purpose \\
\hline SLA 004 & Small & Round & Determinate & Cluster & Cherry type \\
\hline SLA 005 & Small & Round & Indeterminate & Cluster & Cherry type \\
\hline SLA 006 & Small & Round & Indeterminate & Cluster & Cherry type \\
\hline SLA 007 & Medium & Flat round & Determinate & Cluster & Table purpose \\
\hline SLA 008 & Small & Round & Indeterminate & $\begin{array}{c}\text { Cluster } \\
\text { (long) }\end{array}$ & Cherry type \\
\hline SLA 009 & Medium & Round & Determinate & Cluster & Table purpose \\
\hline SLA 010 & Big & Flat round & Semi Determinate & Cluster & Table purpose \\
\hline SLA 011 & Small & Round & Indeterminate & Cluster & Cherry type \\
\hline SLA 012 & Small & Round & Indeterminate & $\begin{array}{c}\text { Cluster } \\
\text { (long) }\end{array}$ & Cherry type \\
\hline
\end{tabular}




\section{Continued}

\begin{tabular}{|c|c|c|c|c|c|}
\hline SLA 013 & Small & Round & Indeterminate & $\begin{array}{l}\text { Cluster } \\
\text { (long) }\end{array}$ & Cherry type \\
\hline SLA 014 & Medium & Round & Determinate & Cluster & Table purpose \\
\hline SLA 015-1 & Small & Round & Indeterminate & $\begin{array}{c}\text { Cluster } \\
\text { (long) }\end{array}$ & Cherry type \\
\hline SLA 015-2 & Small & Round & Indeterminate & $\begin{array}{c}\text { Cluster } \\
\text { (long) }\end{array}$ & Cherry type \\
\hline SLA 015-3 & Small & Round & Indeterminate & $\begin{array}{c}\text { Cluster } \\
\text { (long) }\end{array}$ & Cherry type \\
\hline SLA 018 & Medium & Round & Semi Determinate & Cluster & Table purpose \\
\hline SLA 025-1 & Medium & Round & Semi Determinate & Cluster & Table purpose \\
\hline SLA 025-2 & Medium & Round & Semi Determinate & Cluster & Table purpose \\
\hline SLA 025-3 & Medium & Round & Semi Determinate & Cluster & Table purpose \\
\hline SLA 025-4 & Medium & Round & Semi Determinate & Cluster & Table purpose \\
\hline SLA 025-5 & Medium & Round & Semi Determinate & Cluster & Table purpose \\
\hline SLA 025-6 & Medium & Round & Semi Determinate & Cluster & Table purpose \\
\hline SLA 025-7 & Medium & Round & Semi Determinate & Cluster & Table purpose \\
\hline FOBHT8 & Medium & Round & Determinate & Cluster & Table purpose \\
\hline MOBHT8 & Medium & Round & Determinate & Cluster & Table purpose \\
\hline BARI Tomato-2 & Medium & Round & Determinate & Cluster & Table purpose \\
\hline BARI Tomato-11 & Small & Oval & Indeterminate & Cluster & Cherry type \\
\hline BARI Tomato-14 & Medium & Round & Determinate & Cluster & Table purpose \\
\hline BARI Tomato-15 & Medium & Oval & Determinate & Cluster & Table purpose \\
\hline BARI Tomato-16 & Medium & Round & Determinate & Cluster & Table purpose \\
\hline BARI Tomato-17 & Big & Flat round & Determinate & Cluster & Table purpose \\
\hline BARI Tomato-18 & Medium & Round & Determinate & Cluster & Table purpose \\
\hline BARI Tomato-19 & Medium & Oval & Determinate & Cluster & Processing type \\
\hline BARI Tomato 20 & Medium & Round & Indeterminate & Cluster & Cherry type \\
\hline BARI Tomato 21 & Medium & Oval & Semi Determinate & Cluster & Table purpose \\
\hline
\end{tabular}

it was revealed that all the entries showed different level of resistance or susceptibility against late blight infection (\%), TYLCV infection (\%), bacterial wilt infection (\%), leaf miner infestation (\%), fruit borer infestation (\%) (Table 3).

In case of late blight infection, the range of infection was $0 \%$ to $53 \%$ at later stage of growing condition. The zero percent infection was observed in 34 entries, while 50\% - 53\% infection was observed in 10 entries. The TYLCV infection was is an important disease for the tomato cultivation and it was observed $0 \%$ to $27 \%$ infection, while 47 entries showed $0 \%$ and 11 entries showed $20 \%$ $27 \%$ infection. Bacterial wilt infection was very negligible among the entries in 
the study, where the range was $0 \%$ to $10 \%$ and zero percent infection was observed in 63 entries. Since the season was winter season, that is why the bacterial wilt infection was low.

In case of leaf miner, $0 \%$ to $43 \%$ infestation was observed among the entries. Zero percent infestation was observed in 26 entries, while more than $25 \%$ infestation was observed on 16 entries. The most devastating insect was fruit borer in this study, while the infestation range was $0 \%$ to $10 \%$. The zero percent infestation was observed in 43 entries, while 10\% infestation was observed in 3 entries.

In terms of fruit size, fruit shape, plant growth nature, cluster nature of fruit, type of fruit characters of the tomato entries, there was a wide variation was observed (Table 4). Three sizes of fruits were observed viz., big (8 entries), medium (55 entries) and small (12 entries), while round type was observed in 47 entries, oval was in 16 entries and flat round was in 12 entries. Three types of plant were observed viz., determinate (46 entries), semi-determinate (14 entries), indeterminate (15 entries). Though all the fruits were beard cluster orientation but medium cluster was in 67 entries, while long type cluster was in 8 entries. Maximum tomato fruits were table purpose (58 entries), while cherry type was produced in 16 entries and one was processing type.

\section{Conclusion}

The TYLCV infection was observed $0 \%$ to $27 \%$ infection, while 47 entries showed zero percent infection. The range of bacterial wilt infection was $0 \%$ to $10 \%$ and zero percent infection was observed in 62 entries. In case of leaf miner infestation and fruit borer infestation the range was $0 \%$ to $43 \%$ and $0 \%$ to $10 \%$, respectively. Considering tolerance to late blight, TYLCV, bacterial wilt infection and leaf miner, fruit borer infestation, fruit size, fruit shape, plant growth nature, cluster nature of fruit, type of fruit ten entries AVTO 1010, AVTO 1706, AVTO 1713, AVTO 1829, AVTO 1909, AVTO 1911, AVTO 1915, AVTO 1921, AVTO 1954 and SLA 011 were found zero percent late blight, TYLCV, bacterial wilt infection and leaf miner, fruit borer infestation. So, these ten entries can be selected for disease and insect tolerant tomato varieties development as well as developing disease and insect tolerant hybrid tomato varieties.

\section{Acknowledgements}

This study was completed with the financial support of AFACI under the project of "Development of vegetable varieties in Asia Region".

\section{Conflicts of Interest}

The authors declare no conflicts of interest regarding the publication of this paper.

\section{References}

[1] Agarwal, S. and Rao, A.V. (1998) Tomato Lycopene and Low Density Lipoprotein Oxidation: A Human Dietary Intervention Study. Lipids, 33, 981-984. 
https://doi.org/10.1007/s11745-998-0295-6

[2] Erdman, J.W., Ford, N.A. and Lindshield, B.L. (2009) Are the Health Attributes of Lycopene Related to Its Antioxidant Function. Archives of Biochemistry and Biophysics, 483, 229-235. https://doi.org/10.1016/j.abb.2008.10.022

[3] Prakash, A. and Kumar, A. (2014) Implicating the Role of Lycopene in Restoration of Mitochondrial Enzymes and BDNF Levels in $\beta$-Amyloid Induced Alzheimer's Disease. European Journal of Pharmacology, 741, 104-111. https://doi.org/10.1016/j.ejphar.2014.07.036

[4] Giovannucci, E. (1999) Tomatoes, Tomato-Based Products, Lycopene, and Cancer: Review of the Epidemiologic Literature. Journal of the National Cancer Institute, 91, 317-331. https://doi.org/10.1093/jnci/91.4.317

[5] Balestrieri, M.L., De Prisco, R., Nicolaus, B., Pari, P., Moriello, V.S., Strazzullo, G., Iorio, E.L., Servillo, L. and Balestrieri, C. (2004) Lycopene in Association with R-Tocopherol or Tomato Lipophilic Extracts Enhances Acyl-Platelet-Activating Factor Biosynthesis in Endothelial Cells during Oxidative Stress. Free Radical Biology and Medicine, 36, 1058-1067. https://doi.org/10.1016/j.freeradbiomed.2004.01.014

[6] Di Mascio, P., Kaiser, S. and Sies, H. (1989) Lycopene as the Most Efficient Biological Carotenoid Singlet Oxygen Quencher. Archives of Biochemistry and Biophysics, 274, 532-538. https://doi.org/10.1016/0003-9861(89)90467-0

[7] Stahl, W. and Sies, H. (1996) Lycopene: A Biologically Important Carotenoid for Humans. Archives of Biochemistry and Biophysics, 336, 1-9. https://doi.org/10.1006/abbi.1996.0525

[8] Clinton, S.K. (1998) Lycopene: Chemistry, Biology and Implications for Human Health and Disease. Nutrition Reviews, 56, 35-51. https://doi.org/10.1111/j.1753-4887.1998.tb01691.x

[9] Kotíková, Z., Lachman, J., Hejtmánková, A. and Hejtmánková, K. (2011) Determination of Antioxidant Activity and Antioxidant Content in Tomato Varieties and Evaluation of Mutual Interactions between Antioxidants. LWT-Food Science and Technology, 44, 1703-1710. https://doi.org/10.1016/j.lwt.2011.03.015

[10] Kennedy, G.G. (2003) Tomato, Pests, Parasitoids and Predators: Tritrophic Interactions Involving the Genus Lycopersicon. Annual Review of Entomology, 48, 51-72. https://doi.org/10.1146/annurev.ento.48.091801.112733

[11] Singh, D., Singh, S., Cheema, D.S. and Brar, K.S. (1994) Resistance to Helicoverpa armigera (Hubner) in Tomato Genotypes. Journal of Insect Science, 7, 28-31.

[12] Brar, K.S., Raman, H., Dhaliwal, H.S. and Cheema, D.S. (1995) Field Screening of Different Species of Lycopersicon against Tomato Fruit Borer, Helicoverpa armigera (Hubner). Journal of Insect Science, 8, 196-197.

[13] Kalloo, G. (1991) Genetic Improvement of Tomato. Monographs on Theoretical and Applied Genetics, No. 14. Springer-Verlag, Berlin.

https://doi.org/10.1007/978-3-642-84275-7

[14] Brewer, M.J., Trumble, J.T., Alvarado-Rodriguez, B. and Chaney, W.E. (1990) Beet Armyworm (Lepidoptera: Noctuidae) Adult and Larval Susceptibility Three Insecticides in Managed Habitats and Relationship to Laboratory Selection of Resistance. Journal of Economic Entomology, 83, 2136-2146. https://doi.org/10.1093/jee/83.6.2136

[15] Oatman, E.R. and Kennedy, G.G. (1976) Methomyl-Induced Outbreak of Liriomyza sativa on Tomato. Journal of Economic Entomology, 69, 667-668.

https://doi.org/10.1093/jee/69.5.667 
[16] Saberfar, F. and Sheikhi, G. (2009) Influence of Phenological and Physical Characters of Tomato on Fruit Borer, Helicoverpa armigera (Hubner) Infestation. Resis tant Pest Management Newsletter, 19, 46-47.

[17] Yurk, B. and Powell, J. (2010) Modeling the Effects of Developmental Variation on Insect Phenology. Bulletin of Mathematical Biology, 72, 1334-1360. https://doi.org/10.1007/s11538-009-9494-7

[18] Waluniba and Alemla Ao, A. (2014) Seasonal Incidence of Insect-Pests in Tomato (Lycopersicon esculantum M.) on Different Planting Dates and Its Correlation with Abiotic Factors. International Journal of Bio-Resource and Stress Management, 5 , 280-284. https://doi.org/10.5958/0976-4038.2014.00568.5 